\title{
La lumière au bout du tunnel, ou comment concentrer et séparer des microalgues en combinant lumière et écoulement
}

Salima Rafaï (salima.rafai@univ-grenoble-alpes.fr) et Philippe Peyla

Laboratoire Interdisciplinaire de Physique (UMR5588 CNRS-Université Grenoble Alpes), Domaine Universitaire, BP 87, 38402 St Martin d'Hères Cedex
La microalgue verte

Chlamydomonas reinhardtii

(Chlamy) est capable

de se déplacer au sein de son

environnement liquide à l'aide de ses deux flagelles. Chlamy

possède un " stigma » - l'œil le

plus simple du monde biologique

- qui lui permet d'être phototactique,

c'est-à-dire de pouvoir se diriger

vers une source lumineuse.

Nous décrirons ici le phénomène

de photofocalisation par lequel,

grâce à un écoulement et

de la lumière, les microalgues

sont amenées à se concentrer

spontanément au centre d'un

canal. En outre, nous montrerons

comment les propriétés

de ce micronageur peuvent être

généralisées à d'autres systèmes

et ainsi contribuer à une

compréhension plus globale

des écoulements de matière active.

\section{La nage de Chlamy}

Chlamy (photo p. 26), ce micro-organisme unicellulaire, utilise ses deux flagelles pour produire un mouvement qui ressemble à une brasse ultrarapide (plusieurs dizaines de battements par seconde). Son diamètre de dix micromètres en fait ce qu'on appelle un micronageur. Des observations au microscope, couplées à de l'analyse d'images et du suivi des particules, permettent de montrer que Chlamy adopte une marche aléatoire avec persistance (voir l'article d'introduction), avec une vitesse de l'ordre d'une centaine de microns (soit dix fois sa taille) par seconde [1, 2]. Environ toutes les cinq secondes (temps de persistance) et en l'absence de lumière, Chlamy change de direction aléatoirement.

Il est intéressant de noter que ce mode de nage est commun à de nombreux micronageurs comme par exemple les bactéries, les globules blancs, les spermatozoïdes ou même les nageurs artificiels fabriqués à partir de colloïdes actifs ; ceci fait de Chlamy un système représentatif de toute une classe de micronageurs.

À l'instar de la bactérie Escherichia coli (E. coli), Chlamy est un système très bien caractérisé par les biologistes et présente des particularités intéressantes par rapport à son homologue procaryote. Chlamy possède une forme sphérique simple à modéliser, sa taille dix fois plus importante la rend insensible aux fluctuations thermiques et, enfin, les systèmes de propulsion de ces deux micronageurs sont opposés. Alors que Chlamy tire le fluide devant elle pour avancer, E. coli le pousse à l'arrière (voir introduction). Ainsi, ces deux systèmes aux particularités complémentaires représentent deux micronageurs du monde vivant servant de modèles aux différentes communautés de scientifiques qui s'attachent à étudier la matière active.

\section{Le phototactisme}

Chlamydomonas reinhardtii possède un "stigma » sur le côté de la cellule. Il s'agit d'un photorécepteur qui représente "l'œil " le plus simple du monde biologique. Une fois un signal lumineux capté par le stigma, une cascade biochimique s'ensuit au sein de la cellule et induit une modification des battements de flagelles qui mène finalement à une réorientation de la microalgue vers la source lumineuse. Ce « capteur photosensible " permet ainsi à Chlamy d'orienter sa nage vers une source lumineuse. Cette propriété, appelée phototactisme (positif), permet à la cellule de se déplacer vers des zones optimales pour la photosynthèse en journée. Au contraire, durant la phase nocturne de son cycle circadien, le phototactisme négatif permet à la cellule de s'éloigner des éventuelles sources lumineuses. Ainsi, la nage qui était aléatoire en l'absence de lumière devient directionnelle - on dit encore «balistique»- en présence d'une source lumineuse. Autrement dit, le temps de persistance de la nage de Chlamy 


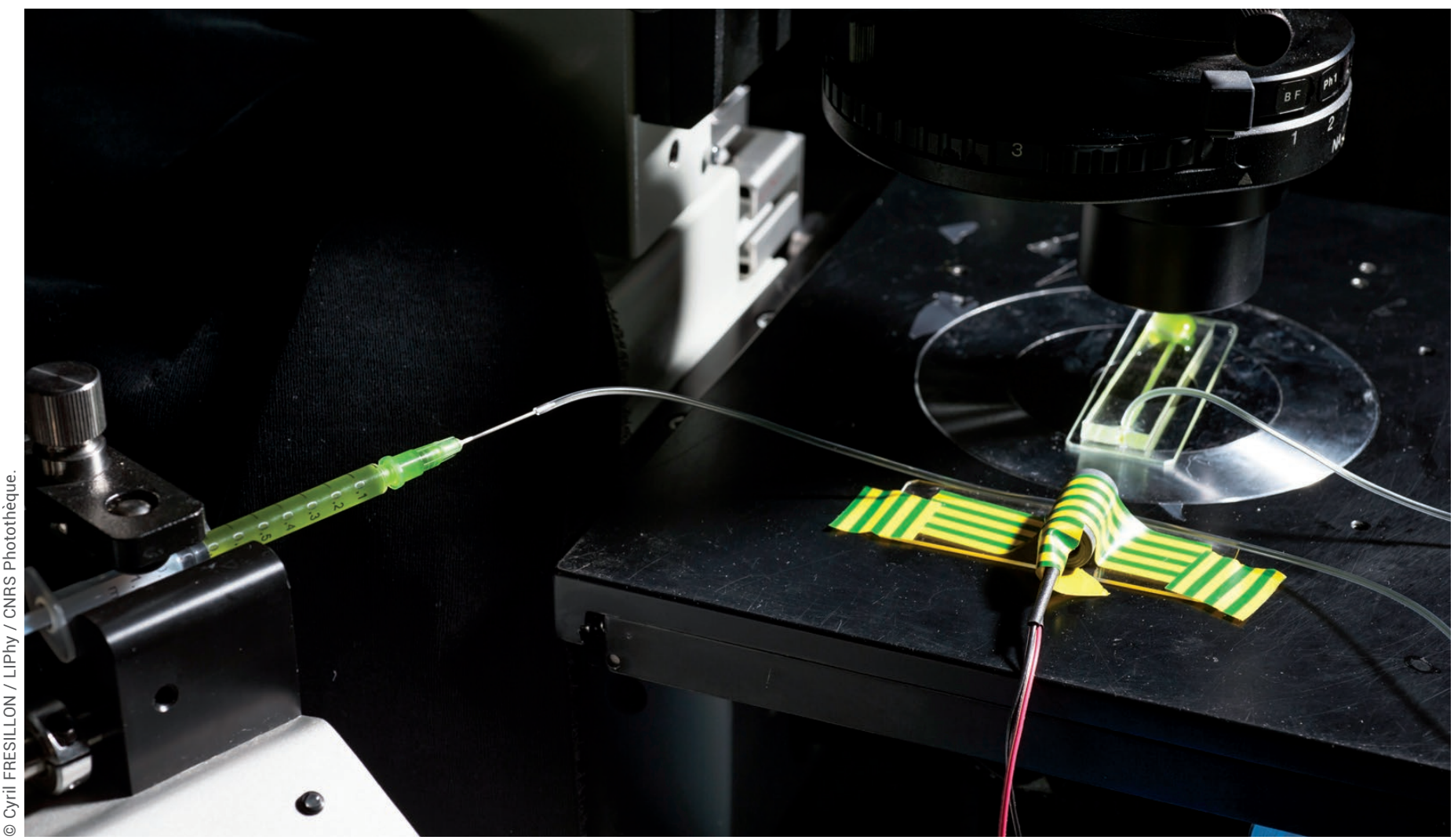

Montage expérimental au laboratoire LIPhy (Grenoble), permettant le couplage entre écoulement et phototactisme pour des suspensions de microalgues Chlamydomonas reinhardtii. À gauche : seringue contenant une suspension active de microalgues. À droite : canal millifluidique pour l'écoulement de cette suspension.

(voir l'article d'introduction) tend vers l'infini en présence de lumière. Le fait d'orienter sa nage dans un gradient quelconque est assez répandu chez les micronageurs du monde vivant. Ces organismes testent périodiquement leur environnement en molécules chimiques, lumière, oxygène, sels ou autres substances, et ensuite traitent l'information à travers un réseau de protéines capables de provoquer la rotation des flagelles.

Le phototactisme ne représente donc qu'un exemple parmi les nombreux "tactismes " présents dans le monde vivant, traduisant la sensibilité des micro-organismes à leur environnement. Il a été démontré que les mécanismes moléculaires de régulation des différents tactismes sont communs. De par le tropisme induit sur le mouvement de Chlamy, le phototactisme peut servir de modèle, dans certaines circonstances, pour tout autre "tactisme " comme le chimiotactisme (nage dans un gradient chimique) ou le gravitotactisme (orientation de la nage dans un champ de gravité).

\section{Photofocalisation}

En soumettant les microalgues à un écoulement en présence d'une source lumineuse en amont du canal d'écoulement, une migration spontanée des micronageurs est observée, conduisant à une autoconcentration des algues au centre du canal (fig. 1). Ce phénomène de photofocalisation [3] peut s'expliquer en couplant l'écoulement dans le canal à la propriété de phototactisme. Un écoulement en canal présente une forme parabolique du profil de vitesse : la vitesse est maximum au centre du canal et nulle au bord (fig. 2a). Une particule dans ce type d'écoulement est sujette à une rotation, car la vitesse du fluide de chaque côté de la particule est différente (fig. 2b). Ainsi, bien que dotée de sa propre vitesse, Chlamy est soumise également à l'écoulement imposé et, en particulier, à une rotation. En effet, la vitesse de l'écoulement varie selon la position entre les deux parois du canal. Ces gradients de vitesse transverses induisent une vorticité qui tend à mettre en rotation tout objet placé dans cet écoulement. En l'absence de source lumineuse, ce simple couplage nage/écoulement mène à des trajectoires oscillantes de Chlamy. En présence d'une

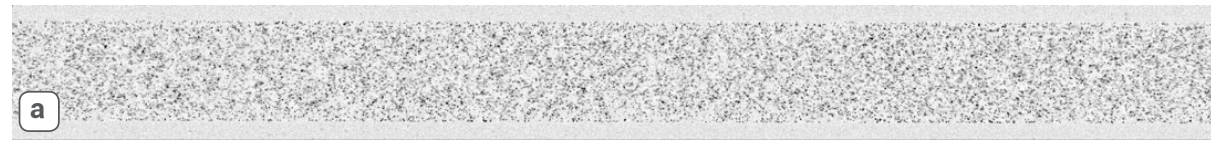

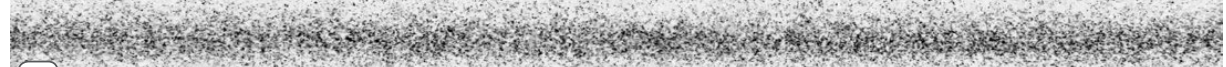
(b)

1. Photofocalisation de microalgues. Les micronageurs sont transportés dans un canal millifluidique de largeur $1 \mathrm{~mm}$. Alors que leur distribution est homogène en l'absence de source lumineuse (a), on observe une focalisation des microalgues au centre du canal en présence de lumière en amont (b). Les dimensions du canal sur ces images sont $1 \mathrm{~mm} \times 9 \mathrm{~mm}$. 


\section{$\gg>$}

source lumineuse en amont de l'écoulement, les cellules tendent à s'orienter vers cette source. Cette orientation privilégiée vers l'amont, couplée à la vorticité de l'écoulement, a pour effet que les algues se dirigent vers le centre du canal (fig. 2c). À l'extinction de la lumière, les algues se dispersent à nouveau dans le canal. Le phénomène est donc parfaitement réversible.

En se basant sur ce simple couplage (phototactisme/vorticité), nous avons proposé une modélisation du phénomène de focalisation qui a permis une bonne description de l'expérience. En particulier, la distribution de densité de micronageurs dans le canal est prédite quantitativement, ainsi que sa variation avec la vitesse imposée de l'écoulement. Les ingrédients retenus pour la modélisation sont très génériques [4] : il s'agit de superposer l'écoulement imposé dans le canal avec la dynamique de nage de type marche aléatoire avec persistance biaisée par un tactisme. La perturbation de l'écoulement par la présence des cellules a été négligée. Ce caractère générique du modèle nous permet de présager une relative universalité du phénomène. C'est d'ailleurs le même mécanisme qui a été observé pour des microalgues gravitotactiques soumises à un écoulement en canal vertical descendant. La photofocalisation des microalgues pourra-t-elle, par exemple, éclairer les mécanismes à l'œuvre dans la réponse immunitaire pour laquelle des cellules motiles, les globules blancs, doivent se frayer un chemin vers les pathogènes en suivant un gradient chimique tout en étant transportées par l'écoulement sanguin à grande vitesse ?

\section{Chlamy, un micronageur modèle ?}

L'expérience précédente illustre parfaitement bien la démarche que nous adoptons. D'une part, nous avons montré que la nage de Chlamy peut être décrite par un modèle assez générique de marche aléatoire avec persistance. Ainsi, Chlamy peut être considérée comme un système représentatif de toute une classe de micronageurs. D'autre part, alors que la photofocalisation est un phénomène qui peut sembler très spécifique à Chlamy, la modélisation que nous en proposons montre que les ingrédients minimaux nécessaires sont en réalité très généraux et peuvent s'appliquer à des systèmes de micronageurs différents.

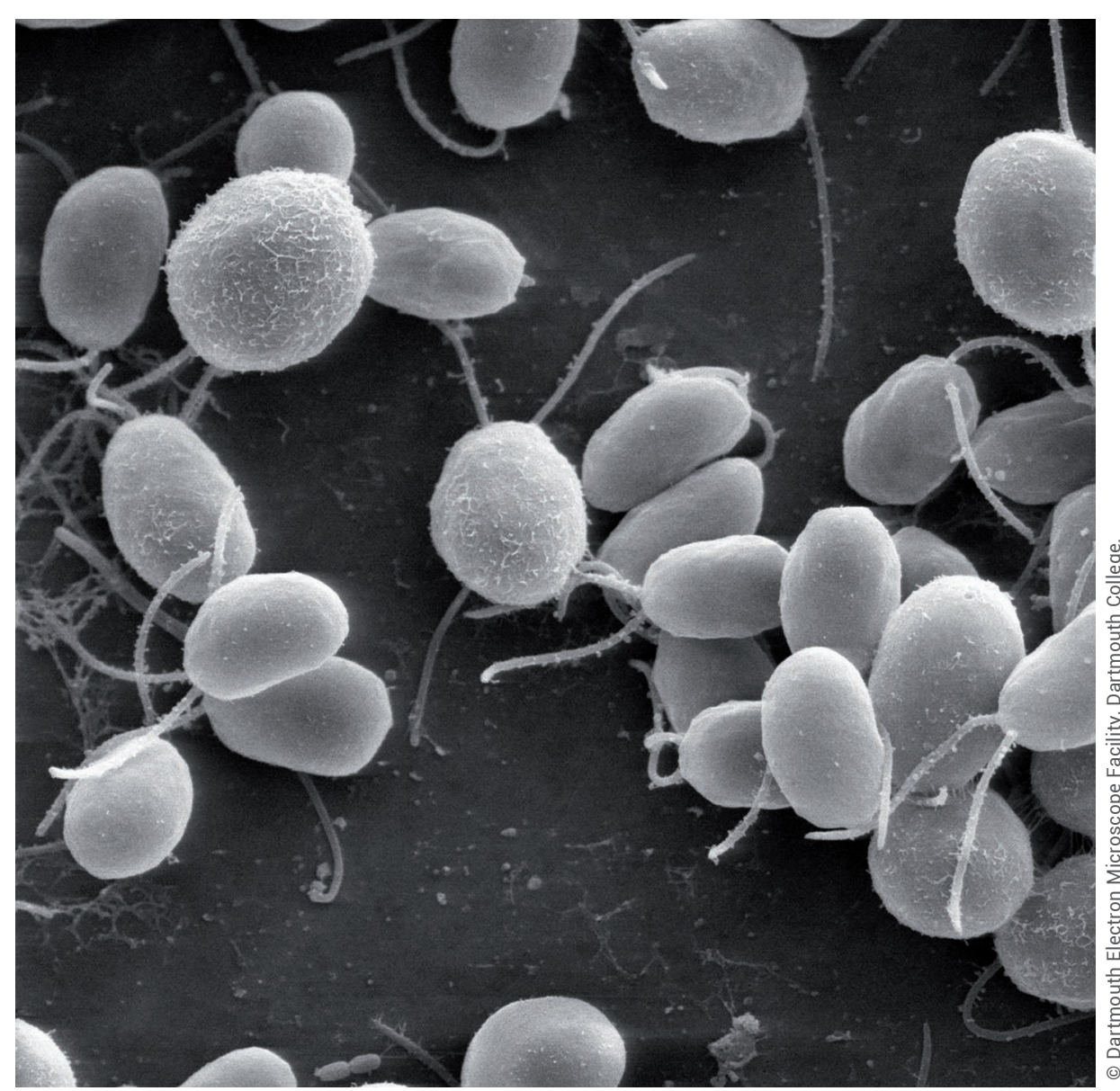

Microalgues Chlamydomonas reinhardtii, observées en microscopie électronique à balayage.
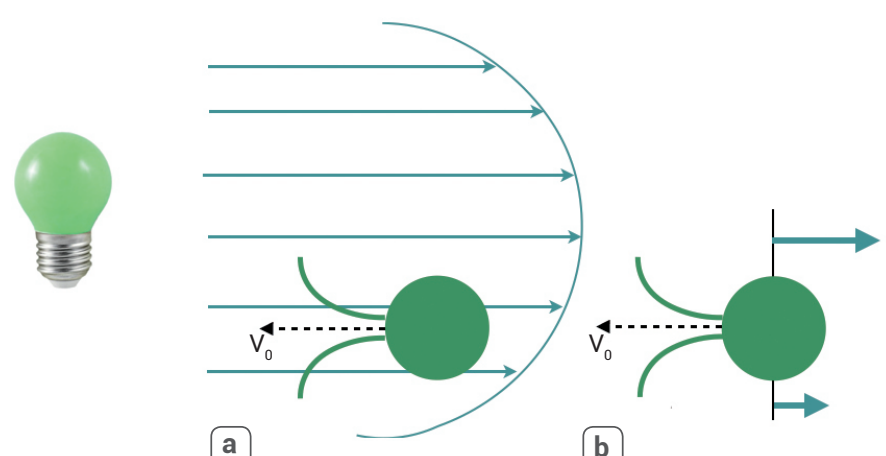

b

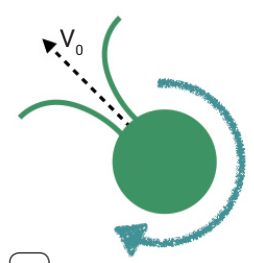

c

2. Schéma descriptif du mécanisme de photofocalisation. Une source lumineuse est placée en amont de l'écoulement parabolique (matérialisé ici par les vecteurs vitesses en bleu). De ce fait, une microalgue, bien que transportée par l'écoulement, présente une orientation privilégiée vers la source lumineuse (a). Elle est également soumise à une rotation due à l'écoulement (b) et, dotée de sa vitesse $\mathrm{V}_{0}$, elle finit par migrer vers le centre du canal (c).

\section{Références}

1• M. Polin, I. Tuval, K. Drescher, J. P. Gollub et R. E. Goldstein, "Chlamydomonas swims with two 'gears' in a eukaryotic version of run-and-tumble locomotion", Science, 325 (2009) 487-490.

2• M. Garcia, S. Berti, P. Peyla et S. Rafaï, "Random walk of a swimmer in a low-Reynolds-number medium", Physical Review E, 83 (2011) 035301 (R).
3• X. Garcia, S. Rafaï et P. Peyla, "Light control of the flow of phototactic microswimmer suspensions", Physical Review Letters, 110 (2013) 138106.

4. M. Martin, A. Barzyk, E. Bertin, P. Peyla et S. Rafaï, "Photofocusing: light and flow of phototactic microswimmer suspension", Physical Review E, 93 (2016) 051101 (R). 